\title{
The Bad Things We Do because of Empathy
}

\section{Fritz Breithaupt}

Germanic Studies, Cognitive Science, Indiana University, Bloomington, IN, USA

Breithaupt, Fritz. (2018). The bad things we do because of empathy. Interdisciplinary Science Reviews, 43(2), 166-174.

\begin{abstract}
Empathy is usually seen as a pro-social and morally positive influence on behaviour. This article provides an overview of cases of negative acts motivated by empathy. The article includes discussions of polarizations that are fueled by side-taking and empathy, selfish forms of empathy (such as sadistic empathy, vampirism, and helicopter parenting), and filtered empathy (using identification with a third person as a medium to have empathy with another). The definition of empathy used is to coexperience the situation of another.
\end{abstract}

Keywords: empathy, dark sides of empathy, sadistic empathy, helicopter parenting, side taking

This article summarizes some of the objections to the understanding of empathy as a morally positive influence on behavior. For several decades, the positive influences of empathy have been the focus of attention and research. In fact, empathy has often been described as one of the key human factors for bettering the planet (Pinker 2011; Nussbaum 2015; Hunt 2008). And there is evidence for the Empathy-Altruism Hypothesis that links empathy with positive prosocial behavior (Batson 2012), though the correlation might be only moderate (Hoffman 2001). People, and some other animals, spontaneously help others when they perceive the other's need or share their feelings.

This article does not want to reject the positive evidence but instead flank it with evidence for negative behavior that results from empathy. The overall point is not to reject empathy as a common or potentially desirable basis for behavior, including positive prosocial behavior, but rather to disconnect empathy from morality in order to align it more closely with aesthetic perception. 
The definition of empathy used in the following is co-experiencing the situation of another. In practical terms, this definition partially overlaps with the often used presented idea of empathy as emotion sharing or affect-sharing (Preston \& de Waal, 2001; Lamm et al., 2017) and models based on perception-action coupling that suggest that observing an emotion in another activates the neural mechanisms in an observer responsible for the production of a similar emotion, with an awareness of the difference between self and other (De Vignemont \& Singer, 2006; Decety \& Lamm, 2006). The definition of co-experiencing also emphasizes the awareness of a difference between self and other. However, in co-experiencing there is also emphasis on a qualitative difference of the experience, since co-experiencing involves an outside perception from which the situation of the other appears different than it does from within.

The focus on emotion sharing has the advantage that it can be measured empirically and directly with brain imaging processes. At the same time, the standard of emotion sharing seems to apply to only a few strong emotions and feelings, such as pain, suffering, disgust and a few social affects such as embarrassment, pride, and guilt. Other complex and social emotions, such as love, and mixed emotions present a challenge. Also many relevant states of another person/individual that are not primarily focused on an emotion but involve intensity fall outside of this definition but seem nevertheless relevant for empathy. These could include situations in which another has to make an important decision, is planning for the future, or is unaware of his or her situation. Empathetic observers can understand, feel, and relate to these emotions but do not simply "share" them and they can relate to situations, such as acts of decision-making. Here the definition of co-experiencing the situation of another may offer a better fit. The notion of co-experiencing also emphasizes that we are not simply passive recipients of the situations of others, but active creators and co-creators of the real or imagined situations of others (see Paulus et al, 2013). However, it turns out that for the matters discussed in the following, the difference between these two definitions of empathy is not decisive (for more distinctions of different forms and concepts of empathy, see Batson 2009; Leiberg \& Anders 2006). For further differentiations between Theory of Mind and empathy, see Kanske (2018, in this volume).

Instead of engaging in a methodological discussion or a discussion of concepts, this article wishes to provide an overview of some of the negative acts that are a direct result of empathy. More specifically, this article will focus on the bad things we do because of empathy. In doing so, the article gives a synopsis of some of the arguments I make in my book The Dark Sides of Empathy (Breithaupt 2017; Breithaupt 2018).

\section{Spotlight Vision}

In recent years, a few critical voices have raised objections against empathy. Prominently, Jesse Prinz and Paul Bloom have come forward with opposition to empathy (Prinz 2011; Bloom 2016). Both argue that empathy is not well-suited to support ethically correct decisions. Bloom describes empathy as a means of focusing 
attention for a short time on an individual fate while neglecting the larger picture, longterm solutions, and large numbers of people. We help the person who draws attention to herself or himself for only a brief period. This fits within paradigms of the EmpathyAltruism-Hypothesis, but it does not lead to optimally good behavior since it bypasses others who might need more help and does not necessarily lead to long-term solutions. This line of reasoning leads Bloom to champion reflected behavior and "rational compassion," instead of emotional empathy. Whereas Jesse Prinz does not base his argumentation on psychological data, he comes to similar conclusions in so far as he finds many substantial exceptions to the idea that empathy supports sound ethical decision-making.

Bloom's spotlight effect of empathy heightens attention for special cases. It explains how empathy can be a powerful trigger of behavior since it draws one's awareness to one scene or person. However, it also explains how empathy can be manipulated. People are willing to donate time and money to one hungry child in a commercial, but will not be moved by the fate of thousands who are subject to famine or civil war.

In an extension of Bloom's argumentation, one can add that this effect of empathy can obscure judgment and lead bystanders, including jury members, to side not with the morally correct side, but instead with the person who is good at drawing empathy to himself or herself (Breithaupt 2012). This effect complicates the idea that morality unites bystanders (DeScioli \& Kurzban, 2013).

\section{Polarizing Effects of Empathy}

Connected to the previous point is the idea that empathy can deepen divides, rather than bridge them. Terrorists may feel empathy for people in a conflict to such a degree that they act violently against the other side. Empathy here could be a motivator for murder. In general, when someone is drawn to empathize with people, this can lead him or her to agree to their opinions, share their emotions, and adopt their viewpoints. In situations of conflict, people thereby may support the side they have felt empathy for, while neglecting the other side. If the empathizer adopts some of the preferences of the target of his empathy, it is likely that aversions toward the other side are also adopted. The us-them divide can receive fuel from empathy when it goes beyond the small circle of family and friends (Green 2014).

Social conflicts can function as a powerful trigger of empathy. Human beings are known to make judgments and to take sides quickly (Todorov et al, 2009). Situations of conflict are especially powerful social scenes that draw bystanders in to contemplate the conflict. Unlike most other animals, human beings tend to intervene and therefore often to tame or decide conflicts (Kurzban, DeScioli, \& O'Brien, 2007). In short, social conflicts may be a common trigger for one-sided empathy that can lead to the adoption of negative opinions about the other side. 
This intervention by bystanders may be linked to a dynamic that includes empathy as a motivator for action. Again, it should be noted how quickly people tend to choose a side, often in fractions of a second. The quick judgment alone may not in and of itself be enough for observers to intervene or take a firm stand. The initial quick side-taking may require reinforcement via empathy. Empathy emerges in two steps here - first as a perspective taking and then as emotion sharing. When one vicariously shares the perspective of a person, the situation of the other becomes clearer and more focused. It becomes perceptible to which pressures the other person is reacting. Consequently, the perspective-taker can develop emotions that would fit the perspective they have taken and thus develop empathy by co-experiencing the situation of the other (for other cases of interaction between Theory of Mind and empathy in complex social situations, see Kanske [2018], in this volume). Once empathy develops, this enforces the side-taking dynamic.

From the perspective of the chosen side, the other party of a conflict tends to appear unlikeable, unfair, undesirable, wrong, or bad. The stronger the support one feels for one's chosen side and the stronger the empathy, the more negative the other side appears to be.

The outcome of this dynamic, the Three-Person Model of Empathy (Breithaupt 2012), can lead to people committing aggressive acts against the other side of a conflict. Because they empathize with the one side of the conflict, the other side can be painted in stark negative colors. It is often assumed that empathy has a positive impact on peace negotiations and contributes to a smoothing of tensions. However, the conflictincreasing influence of this side-taking dynamic should not be neglected. Considerations of side-taking and empathy can help to revise understandings of some hateful acts. As mentioned above, some acts of terrorism could be motivated by empathy with the one side and negative hatred of the other side that is deemed to suppress one's chosen side.

\section{Selfishness of Empathy}

An implicit assumption of empathy research and general interest in empathy has been that empathy benefits the target of empathy. There are certainly cases where this happens. Parents tending to children and humanitarian aid seem likely cases of empathy supporting the person in need. We will come to possible objections to both of these examples later. However, the assumption that empathy benefits the target of empathy might have led people to neglect the party that benefits more directly, namely the empathizer himself or herself.

Empathy is quite rewarding for the empathizer. There is evidence that the arousal of emotions is generally perceived as more positive than negative, and that positive emotions play an asymmetrically stronger role in life satisfaction than negative ones (for 
example, Cohn et al, 2011). Empathy also enriches one's range of experiences by expanding the perception of a person beyond his or her own senses and his or her immediate situations. Empathy, transport (Green, Brock, \& Kaufman, 2004), and mobile or mediated consciousness allow people to participate in the lives of others or situations other than their current situation. Humans have the ability to imagine the situations of others or situations other than their current one, and thus feel what one feels in another situation or as someone else.

Researchers are far from understanding the mechanisms of this mobile consciousness, but one can observe some of its effects. One consequence is that we experience more or different things than we would otherwise.

There are numerous highly complex questions connected with these statements. An important question is whether our empathetic experience of others is limited to situations and emotions that we have already experienced in the past. It seems that people acquire general schemas of emotions (pain, embarrassment) and can apply them to specific situations that they have not yet experienced (a broken leg, being fired in a Japanese cultural context). However, it is less clear to which degree this abstraction leads to a loss of intensity and accuracy of empathy.

Another question is where the difference between mere imagination and empathy lies. Many significant brain imaging studies used to determine empathy employ short film clips or narrative accounts of events. They do, indeed, measure immediate brain reactions, but they refer to situations that could be (and actually are often) fictitious accounts containing people or characters the participants did not know before. Hence, it seems that empathy-like processes in fiction and even imagination might structurally be similar to the empathy experienced in the real world.

Empathy's proximity to fiction and imagination might help to explain or reveal the pleasure that is connected to empathy. Perceiving more situations is usually rated as positive. In the case of fiction (film, theater, literature, the arts), even negative situations experienced by the characters seem to be perceived as positive (Menninghaus et al. 2017; Hanich et al., 2014). In the real world, people can derive pleasure from understanding and sharing the feelings of others, even if these are not necessarily positive feelings.

Does this mean that empathy is experienced as rewarding per se? Or is it just the resulting appraisal of situations and the arousing of specific emotions that is perceived as pleasant? The evidence is not clear. Further clues that empathy itself is perceived as rewarding include the proximity of empathy to transportation in fiction and fantasy. In fiction, we might experience something one could describe as empathy without social obligation to the other (Keen 2007), and the experience is generally regarded as intrinsically pleasant. 
In the following, we will discuss several cases in which empathy is perceived as positive by the empathizer, even if the effects are negative for the target of empathy.

\section{Empathic Vampirism}

One of the cases in point for the selfishness of empathy can be called empathic vampirism. The empathizer feels and experiences the world vicariously via others and thereby participates in their fate, without, however, having their best interests in mind. Instead, the implicit interest of the empathizer lies in his or her own act of experiencing. The other becomes a medium of one's experience. Even in cases where the empathizer imagines having the best interests of the other in mind, such as in helicopter parenting, the results may prove otherwise (Schiffrin et al., 2014). In the case of helicopter parents, it could be that the parents' interest lies in experiencing a perfect youth retrospectively via their children, more perfect, at least, than their own.

The name "vampirism" is adopted from Stanley Cavell, who describes vampirism as a response to one's lack of originality, for which one hopes to compensate by drawing from others (Cavell, 2004). In the case of empathy, the basic description of empathic vampirism or selfish empathy consists of the process of sharing another's experience and making it one's own over time, without concern for the other's long-term welfare as an independent being. The force of this definition lies in the idea of making it your own, of appropriating it. In the case of helicopter parents, this lack of differentiation between one's own well-being and the child's well-being can mean neglecting the child's freedom to find a path for himself or herself. Helicopter parents may ignore to consider whether the parent's plan for the child matches the child's ideas for him- or herself.

Stalkers, fans, stage parents, and helicopter parents fall under this definition of using others as medium of their experience. There is not much research on empathy and socalled helicopter parenting, and the definitions of terms are not clear at this point. Transport in fiction can also fall under this definition. All of these vampiristic forms of empathy have in common that the other is ignored as other and becomes subjected to the imperialistic empathizer (a danger that Levinas describes, see Buddeberg [2018], in this volume).

\section{Empathic Sadism}

Another form of selfish empathy can be described as empathic sadism. Empathic sadism, or empathic cruelty in its basic form, means that an empathic observer enjoys the pain or suffering of another (Young 2016). The negative feelings of another are somehow translated by the empathizer into positive feelings for him- or herself. 
Empathic sadism in the broad sense appears in a variety of forms of behavior. It can occur in the enjoyment of tragedies and sad movies (Hanich et al., 2014), as a motivation for punishment (Young 2016), in sadistic acts, in schadenfreude, and in various everyday behaviors like bullying, shaming, and teasing. The differences between these is considerable and includes the degree to which the sharing of emotion occurs and whether the pleasure of the empathizer emerges directly from the sharing of emotions or from an appraisal of that emotion (in punishment, for example, the pain of the other is appraised as positive). It also possible to distinguish the sharing of emotions and vicarious emotions, that are based on non-shared affective experiences (Paulus et al, 2013).

There is also a range of possibilities to explain how positive feelings in the empathetic observer come about. It is possible that some subjects mix up pain and pleasure, that they enjoy the mere state of being moved and aroused, or that they enjoy the fact of being connected with another. The latter cases could be described as empathy for empathy's sake, that is, as the enjoyment of the emotion sharing, even when (or perhaps especially when) the other is suffering. Following this logic, it seems hypothetically possible that a rapist uses empathy to heighten his emotional pleasure since he intensifies the degree of his experience by sharing the high intensity of the victim's suffering.

Sadistic empathy often includes the manipulation of others. Manipulative empathy should be understood as bringing about a situation for the other with the goal of making their emotional response to that situation intelligible, and therefore possible to share. Whereas most forms of manipulative empathy focus on negative emotions, there are some more positive forms, too, such as gift giving.

It is not clear whether so-called psychopaths fall into the description of sadistic empathy. For a long time, the research of psychopathy was dominated by the view that psychopaths lack emotional depth and empathy (see for example, Harenski et al., 2010). More recently, however, brain imaging studies have revealed somewhat reduced but still empathetic patterns exhibited by psychopaths (Meffert et al., 2013; see also Decety et al., 2009). A core difference might be not the lack of empathy capacities, but rather the propensity to use it and not to block it (Keysers \& Gazzola, 2014).

\section{Humanitarian Empathy. Filtered Empathy}

Most proponents of empathy point to situations of humanitarian aid that extend support beyond the simple borders of the we-group. Human beings do help each other in situations of need, and empathy is often named as the likely motivator for this behavior (Brown, 2009). 
However, it is far from clear whether empathy is at work in humanitarian aid and helping behavior. There are other explanations that also fit helping behavior or so-called altruism. One explanation is that morality or religion motivates people to help. Another alternative explanation is more interesting in this context since it also involves an empathy-related mechanism. It states that instead of direct empathy with the person in need, another mental act motivates behavior: identification with the (real or imaginary) helper. The helper is a much more "attractive" target of empathy-identification. Via identification (that is, seeing oneself as oneself in the situation of the other) with the helper, the empathizer participates in the positive aura of the helper.

The identification with the helper still involves some empathy with the person in need, but via the figure of the helper as medium or filter. That means that the person in need only comes into view as a person-in-need, as someone who is a person only in so far as he or she motivates action by the helper. In this respect, this form of empathy can be called indirect, mediated, or filtered.

Is this a dark side of empathy? Does it lead to bad behavior? One could think that as long as this hero-identification leads to prosocial helping behavior beyond the in-group, it should be considered a positive side of empathy. However, there are clear limitations. The hero-helper may expect positive valuations or appreciation for his helping behavior. If the target of empathy fails to deliver this positive validation by thanking the helper, recognizing his or her behavior, or by improving, this can lead to quick dissatisfaction or resentment by the empathizer and to corresponding acts of rejecting and mistreating the other.

Another limitation of the positive side of filtered empathy is that the target of filtered empathy only matters in so far as he or she fits the image of the filter. In the case of humanitarian aid, the target of empathy only matters as the "victim." This can lead to a tendency to maintain or prolong the victim status. For postcolonial critiques of the humanitarian subject along these lines, see Elze (2018), in this volume.

Conclusion

The article briefly discussed a range of negative acts that result from empathy. The listed cases do not provide a conclusive list of negative actions connected to empathy. For example, if one uses a wider definition of empathy to include Theory of Mind, which is sometimes called cognitive empathy and is related to mentalizing, one could add the dark aspects of competition since Theory of Mind can be used to outwit opponents (Bubandt \& Willerslev, 2015). There are also other negative forms of behavior derived 
from empathy, such as belittling others. Empathy also can be harmful to the empathizer by leading to exhaustion or loss of self (Breithaupt 2017, 2018).

Overall, the paper attempts to disconnect empathy from the idea that it is solely morally positive. Considerations of empathy should at least be coupled with considerations of the us-feeling, described by Engelen (2018) in this volume, a mutually relational feeling that includes recognition of the other. This does not mean that empathy should be condemned and that one should be opposed to it. Instead, the argument to be made is that empathy is a powerful way of experiencing the world. Someone who empathizes lives in more than one world and has a richer and more complex perception of the social world. In essence, empathy is a matter of aesthetics more than it is one of morality.

\section{Acknowledgement}

I would like to thank Philipp Kanske and Kai Wiegandt for their dedicated and enthusiastic work, which brought together a diverse group of researchers and artists for the symposium The Fascination with the Unknown: The Other in Leipzig (November 2016). Furthermore, I owe Michael Bryant and the anonymous reviewer gratitude for their thoughtful comments on this paper.

\section{Bibliography}

Batson, Daniel C. "The Empathy-Altruism Hypothesis: Issues and Implications." In: Empathy: From Bench to Bedside, ed. Jean Decety (Cambridge, Mass.: MIT Press, 2012), 41-54.

Batson, Daniel C. ."These Things Called Empathy: Eight Related but Distinct Phenomena," In: The Social Neuroscience of Empathy, ed. Jean Decety (Cambridge, Mass.: MIT Press, 2009), 3-15.

Bloom, Paul. Against Empathy (New York: Harper Collins, 2016).

Breithaupt, Fritz. The Dark Sides of Empathy (forthcoming Ithaca: Cornell UP, 2018).

Breithaupt, Fritz. Die dunklen Seiten der Empathie (Berlin: Suhrkamp, 2017).

Breithaupt, Fritz. “A Three-Person Model of Empathy." In: Emotion Review 4.1 (2012), 84-91.

Brown, Richard. D., ed.. Humanitarianism and Suffering: The Mobilization of Empathy. Cambridge: Cambridge UP, 2009. 
Bubandt, Nils, and Rane Willerslev. "The Dark Side of Empathy: Mimesis, Deception, and the Magic of Alterity." In: Comparative Studies in Society and History 57, no. 1 (2015), 534.

Cavell, Stanley. Cities of Words: Pedagogical Letters on a Register of Moral Life (Cambridge: Belknap Press, 2004).

Cohn, Michael A., Barbara L. Fredrickson, Stephanie L. Brown, Joseph A. Mikels, and Anne M. Conway. "Happiness Unpacked: Positive Emotions Increase Life Satisfaction by Building Resilience." In: Emotion 9, no. 3 (2009), 361-68.

Decety, Jean et al. "Atypical Empathic Responses in Adolescents with Aggressive Conduct Disorder: A Functional MRI Investigation." In: Biological Psychology 80.2 (2009), 203-211.

Decety, Jean, and Claus Lamm. "Human Empathy through the Lens of Social Neuroscience." In: The Scientific World Journal 6 (2006): 1146-1163.

DeScioli, Peter, and Robert Kurzban. "A Solution to the Mysteries of Morality." In: Psychological Bulletin 139, no. 2 (2013), 477-495.

De Vignemont, Frederique, and Tania Singer. "The Empathic Brain: How, When and Why?." In: Trends in Cognitive Sciences 10, no. 10 (2006): 435-441.

Green, Joshua. Moral Tribes: Emotion, Reason, and the Gap Between Us and Them. New York: Penguin, 2014.

Green, Melanie, Timothy C. Brock, and Geoff F. Kaufman. "Understanding Media Enjoyment: The Role of Transportation into Narrative Worlds." In: Communication Theory 14, no. 4 (2004), 311-327.

Hanich, Julian et al. "Why We Like to Watch Sad Films: The Pleasure of being Moved in Aesthetic Experiences." In: Psychology of Aesthetics, Creativity and the Arts 8 (2014), 130-143.

Harenski, C.L. et al. "Aberrant Neural Processing or Moral Violations in Criminal Psychopaths." In: Journal of Abnormal Psychology 21 (2010), 1-12.

Hoffman, Martin L. Empathy and Moral Development: Implications for Caring and Justice. Cambridge: Cambridge University Press, 2001.

Hunt, Lynn. Inventing Human Rights. New York: Norton, 2008.

Keen, Suzanne. Empathy and the Novel. Oxford: Oxford University Press, 2007. 
Keysers, Christian, and Valeria Gazzola. "Dissociating the Ability and Propensity for Empathy." In: Trends in Cognitive Sciences 18, no. 4 (2014), 163-166.

Kurzban, Robert, Peter DeScioli, and Erin O’Brien. “Audience Effects on Moralistic Punishment." In: Evolution and Human Behavior 28.2 (2007), 75-84.

Lamm, Claus, Markus Rütgen, and Isabella C. Wagner. "Imaging Empathy and Prosocial Emotions." In: Neuroscience Letters (2017).

Leiberg, Susanne, and Silke Anders. "The Multiple Facets of Empathy: A Survey of Theory and Evidence." In: Progress in Brain Research 156 (2006), 419-440.

Meffert, Harma, Valeria Gazzola, Johan A. Den Boer, Arnold AJ Bartels, and Christian Keysers. "Reduced Spontaneous but Relatively Normal Deliberate Vicarious Representations in Psychopathy." In: Brain 136, no. 8 (2013), 2550-2562.

Menninghaus, W., Wagner, V., Hanich, J., Wassiliwizky, E., Jacobsen, T., \& Koelsch, S. (2017). The D istancing-E mbracing model of the enjoyment of negative emotions in art reception. Behavioral and Brain Sciences, 1-58.

Nussbaum, Martha C. Political Emotions. Cambridge: Harvard UP, 2015.

Paulus, Frieder M., Laura Müller-Pinzler, Stefan Westermann, and Sören Krach. "On the Distinction of Empathic and Vicarious Emotions." In: Frontiers in Human Neuroscience 7 (2013), 196.

Pinker, Steven. The Better Angels of Our Nature: The Decline of Violence in History and its Causes. London: Penguin, 2011.

Prinz, Jesse. "Against Empathy." In: The Southern Journal of Philosophy 49.1 (2011), 214233.

Schiffrin, Holly H., et al.. "Helping or Hovering? The Effects of Helicopter Parenting on College Students' Well-Being." In: Journal of Child and Family Studies 23.3 (2014), 548557.

Todorov, Alexander, Manish Pakrashi and Nikolaas N. Oosterhof. "Evaluating Faces on Trustworthiness after Minimal Time Exposure." In: Social Cognition 27.6 (2009), 813833.

Young, Allan. "Empathic Cruelty and the Origins of the Social Brain." In: Critical Neuroscience: A Handbook of the Social and Cultural Context of Neuroscience. New York: Wiley-Blackwell, 2016. 
